\title{
Segmenting 3D Branching Tubular Structures Using Cores
}

\author{
Yonatan Fridman, Stephen M. Pizer, Stephen Aylward, and Elizabeth Bullitt \\ Medical Image Display \& Analysis Group \\ University of North Carolina, Chapel Hill, NC \\ fridmanecs. unc.edu
}

\begin{abstract}
Blood vessels and other anatomic objects in the human body can be described as trees of branching tubes. The focus of this paper is the extraction of the branching geometry in $3 \mathrm{D}$, as well as the extraction of the tubes themselves via skeletons computed as cores. Cores are height ridges of a graded measure of medial strength called medialness, which measures how much a given location resembles the middle of an object as indicated by image intensities. The methods presented in this paper are evaluated on synthetic images of branching tubular objects as well as on blood vessels in head MR angiogram data. Results show impressive resistance to noise and the ability to detect branches spanning a variety of widths and branching angles.
\end{abstract}

\section{Introduction}

Three-dimensional medical images are often difficult for a physician to view both due to the noise introduced by the imaging process and because of the problems inherent in visualizing any volume image. Tasks such as surgical planning and guiding a catheter through a blood vessel tree can be facilitated with an accurate segmentation [1]. This paper considers a method for computing such a segmentation using 1D cores of branching tubular objects, such as blood vessels, in 3D images of the quality found in MR angiograms. Cores are medial axes, at scale, computed using methods developed in the Medical Image Display and Analysis group at the University of North Carolina at Chapel Hill [2], [3]. Not only are cores insensitive to image noise and small object boundary perturbations [4], but they are computed directly from image grayscale information, so they can be used as an automated segmentation tool. Cores carry with them radius and orientation information of the object(s) they represent, providing additional geometric information [5].

The method presented can be compared with other methods for the segmentation of tubular objects. Frangi et al. [6] use a model-based method where an object's approximate medial axis is coupled to its boundary and then refined. Lorigo et al. [7] use a second order level set method to rapidly segment a whole image. Vasilevskiy et al. [8] use a geometric flow method in which a surface evolves under image-based constraints so as to cling to object boundaries. Aylward et al. [9] use a multi-scale image intensity ridge traversal method with branch handling. They separately search for position and width information and define orientation implicitly, whereas the ap- 
proach of this paper is to simultaneously determine position, width, and orientation information as a ridge of medialness.

The work presented in this paper is built upon the work of Furst [3] and Aylward et al. [9], [10]. It extends the work of Furst by improving the robustness of his core following and termination and by augmenting his method with the ability to handle branching objects. It differs from the work of Aylward et al. by relying on only one seed point per tree structure and no post-processing to segment a tubular branching object.

Section 2 of this paper describes a predictor-corrector method for marching along $1 \mathrm{D}$ cores of tubular objects in 3D images. Section 3 describes a method for determining when a core of a tubular object reaches a branch in that object. The cores defined in this paper mathematically do not branch [11], so once such a branch in an object is encountered, the method locates and follows the cores of the two new branches. Section 4 introduces a method for terminating core following when an object ends while still managing to traverse small breaks in the object. Sections 5 and 6 provide results and evaluate methods applied both to synthetic images and to clinical images.

\section{Core Following}

Before beginning core following the image to be analyzed is pre-processed using Westin's 3D oriented adaptive filtering technique [12] in order to suppress image noise while enhancing objects of interest. Once the image is filtered in this way, it is segmented using a marching algorithm based on medial atoms and medialness.

Medial Atoms. The method described in this section extracts a sampled core composed of discrete medial atoms. A medial atom $\underline{m}$ in 3D is a structure defined by four parameters $(\underline{\mathrm{x}}, \mathrm{r}, \underline{\mathbf{F}}, \theta)$ - the coordinates of the atom in 3 -space, the radius of the object of interest at location $\underline{x}$, a frame that describes the orientation of the medial atom, and an object angle that describes the rate of widening or narrowing of the object (Fig. 1a). In this paper the object angle is constrained to $\pi / 2$, assuming objects with nearly parallel sides. These four parameters imply a set of concentric vectors, known as spokes, that extend from the medial location $\underline{x}$ to the implied object boundary.

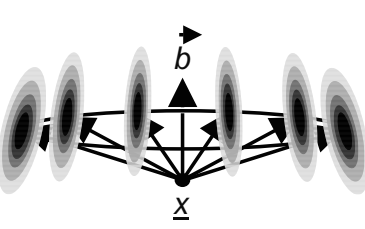

(a)

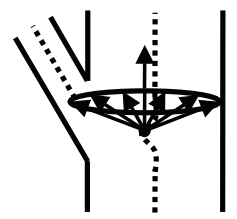

(b)

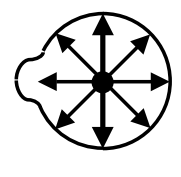

(c)

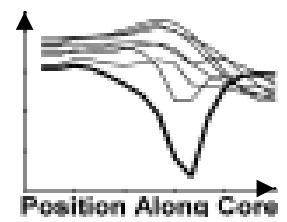

(d)

Fig. 1. (a) The set of concentric spokes of length $r$ that make up a medial atom in 3D. The vector $\mathbf{b}$ is the core tangent direction. Weighting functions are placed at the tip of each spoke. (b) A medial atom at a branch of a tubular object with cores shown as dotted lines. (c) A crosssectional view of the tube and medial atom as a branch starts forming on the left. (d) The spoke at 9 o'clock (curve shown in bold) gives the weakest response near a branch among the eight spokes. This plot shows responses along a section of vessel from the image in Figure 5. 
Medialness. The medialness $\mathrm{M}(\underline{\mathrm{m}})$ of a medial atom is a scalar function that measures the fit of the medial atom to image data. For the purposes of this paper it is measured by placing a derivative of a Gaussian at the tip of each spoke, where the derivative is taken in the direction of the spoke. These functions provide weights on the image and the results of the weighting are integrated to give the medialness value. In $3 \mathrm{D}$, this is given by the equation

$$
\int_{\vec{v} \in V} D_{\hat{\hat{v}}} I(\underline{x}+\vec{v}, \sigma)
$$

where $\mathbf{V}$ is the set of concentric spokes. $\sigma$ is the scale of interrogation, i.e., the standard deviation of the Gaussians. The methods presented in this paper use a scale of interrogation that is constant across object widths. This choice is meant as a compromise between the detection rate and the localization accuracy of medial atoms, since detection is improved by increasing the scale of interrogation while localization is improved by decreasing the scale of interrogation [13].

In the method described here, to select a medial atom as being on the core, medialness is first optimized over the parameter space $(\mathrm{r}, \underline{\mathbf{F}})$. The location $\underline{\mathbf{x}}$ of the medial atom is then found by further optimizing medialness over the spatial plane that is normal to the core tangent and that passes through an estimated position $\underline{\mathbf{x}}_{0}$. This choice of optimization space defines what is known as an optimum parameter core [2], [3]. This method of determining medial atoms is used in a predictor-corrector marching algorithm [14] to extract a core, taking a step from a given medial atom $i$ in the tangent direction of atom $i$ to predict the location of atom $i+1$. Then the location, radius, and orientation of atom $i+1$ are corrected using the described optimization. For a more detailed discussion of cores see [5].

\section{Branch Handling}

The cores defined in this paper do not have branch points. Thus, the core following method attempts first to detect when the core being followed reaches the object's branching area and subsequently to locate the two new branches and to resume following each of the cores of these new branches.

Branch detection is accomplished in two steps. The first step is to apply an affineinvariant corner detector, $\mathrm{L}_{\mathbf{u u}} \mathrm{L}_{\mathbf{v}}$ [15], [16], [17], to the three-dimensional image, where $\mathbf{v}$ is the image gradient direction and $\mathbf{u}$ is the eigenvector corresponding to the largest eigenvalue of the Hessian in the plane normal to $\mathbf{v}$. This operator is applied at the same scale at which medialness is computed (see section 2). This consistently gives strong responses at corners of branches, but also gives strong responses in undesirable places such as the inside edge of a sharply bending object or places that have voxel jaggedness due to noise or aliasing (Fig. 2).

Medial atoms whose spoke tips are at maxima of cornerness are potential branch points. Once these potential branch points are determined the second step is to identify and discard false positives as follows. 


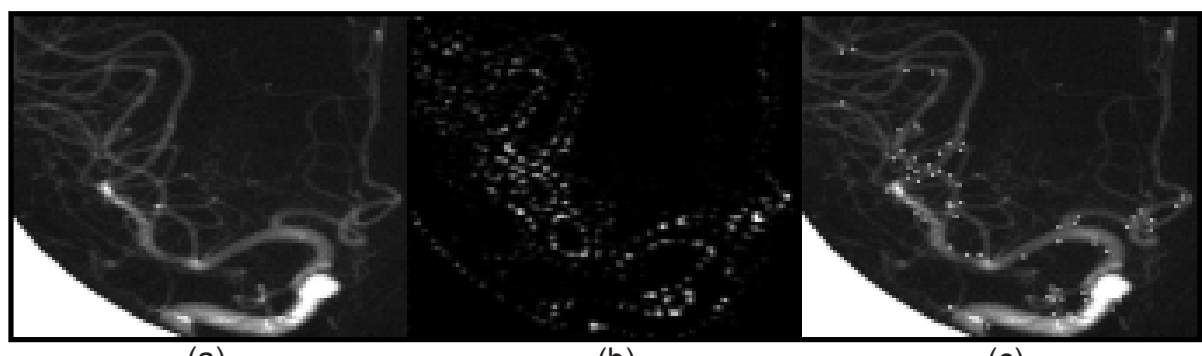

(a)

(b)

(c)

Fig. 2. (a) A 2D DSA projection image of the head. (b) The affine-invariant corner detector $\mathrm{L}_{\mathbf{u u}} \mathrm{L}_{\mathbf{v}}$ applied to the DSA image at a scale of $\boldsymbol{\sigma}=2$ pixels. (c) Local maxima of cornerness (white dots) displayed on the original image, where only maxima above a given (arbitrary) threshold of cornerness are shown. This example is shown in 2D to facilitate visualization; similar results are achieved in $3 \mathrm{D}$.

As a core nears a branch it begins bisecting the branching angle, but then typically snaps back to one of the two children (Fig. 1b). In snapping back to one of the children, the core jumps from a local optimum in medialness at the original core to a stronger optimum at the new core, it does not follow a single core that continues through the branch. Just before this snapping occurs, the responses of one or two spokes decrease dramatically with respect to the rest of the spokes [9] (Fig. 1c, d). If this decrease does not occur, the potential branch is rejected as a false positive. If this decrease does occur, core following must resume at the two child branches.

Since the core automatically snaps to one of the two branches, the algorithm only needs to locate the other branch. As described, the medial atom spoke that lies on the intersection of the two branches gives a significantly weaker response in medialness (see Fig. 1). The location of this spoke tip provides an approximate starting location for the core of the new branch and the direction in which it points provides an approximate tangent direction for the new core. The radius of the new core is estimated as $1 / 2$ times the radius of the original core. Once the new medial atom is estimated it is refined by the corrector portion of the predictor-corrector method. These estimates are sufficiently accurate for the corrector portion of the method to locate the new core, as shown in section 5.1 .

\section{Core Termination}

When following the core of an object such as a blood vessel, the object eventually comes to an implicit end due to a decreased signal-to-noise ratio. The method used for deciding when to terminate core following is based on the confidence with which the desired core is detected, determined by the strength of the medialness value of the core relative to local statistics on the expected medialness value of the core. At each step along the core a large number of randomly positioned and oriented medial atoms are sampled in the spatial vicinity of the core to get a sense for the range of expected medialness values. It is assumed that the large majority of these medial atoms will not be aligned with an object. Due to the way in which medialness is defined in this paper 
(see Eq. 1) medialness can be either positive or negative and the mean medialness value of random medial atoms should be approximately zero. If the mean is not near zero, the image is resampled. If the medial atom along the detected core is more than three standard deviations above the mean then it is accepted as a valid core point, otherwise it is flagged. If at least four out of five consecutive medial atoms are flagged as having weak medialness, core following is terminated.

\section{Results}

The method described was tested in two ways. First, several sets of synthetic images were created to simulate blood vessels. Each set of synthetic images was created to test a different part of the method (core following, branch handling, and termination) and each of these parts of the method was tested separately. Second, the method was tested as a whole on clinical MRA data of the head.

\subsection{Results in Synthetic Images}

The basic core following, without accounting for branching and ending, is quite robust in the presence of image noise. Figure 3 shows one slice of a three-dimensional image of a curved binary tube that has a radius of three voxels. Gaussian noise is added to the image with a magnitude per voxel of approximately five times what would be expected in an MRA [9]. Even in such a noisy image the core of the entire tube was extracted consistently over several test runs. Similar results were achieved for tubes of different sizes, curvatures, and torsions. In images with a level of noise commonly seen in an MRA cores were extracted consistently from tubes with a radius of slightly less than one voxel.

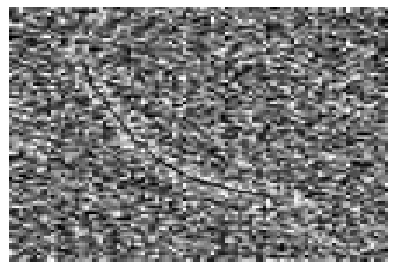

Fig. 3. One slice of a noisy synthetic 3D image of a tube of radius three pixels whose axis lies entirely in the plane displayed. The black curve is the core of the tube.

Branch handling was tested on a set of 36 simulated images of branching tubular structures spanning a range of branching angles, branch sizes, and background noise levels. The code was run on each image ten times. A branching angle of 0 degrees in Figure 4 indicates that the two child branches have axes that share a tangent direction at the point where they branch and which then diverge. The branch size labels indicate the cross-sectional area of the smaller of the two child branches relative to that of the parent, where the cross-sectional areas of the two child branches sum to the cross- 
sectional area of the parent. Three different levels of Gaussian noise were added to the image - low (approximately half of what would be expected in an MRA), normal (approximately what would be expected in an MRA), and high (approximately twice what would be expected in an MRA). The code detected the branch correctly in all 120 test runs with low background noise. Of the 120 runs with normal background noise the branch was missed on three runs, all three of which had a small branch size ( 0.1 times the cross-sectional area of the parent). Of the 120 test runs with high background noise the branch was missed on 24 test runs, 17 of which had a small branch size. The results for the 120 high noise test runs are summarized in Figure 4 . In the cases where the branch point was detected correctly, continuation of core following in the two new branches was accomplished correctly $98 \%$ of the time.

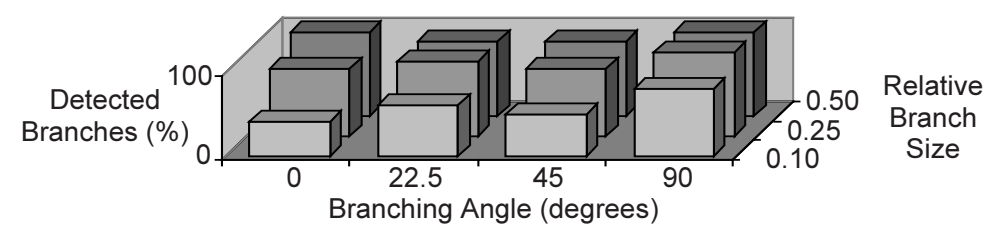

Fig. 4. The success of the branch detection code on synthetic images with a noise level of approximately twice what would be expected in an MRA. Each bar shows the percentage of test runs on which the branch was correctly detected for a given branch angle and a given branch size.

As described in section 4, core following should terminate when there is a significant decrease in the image signal-to-noise ratio. The difficulty is in following the object as long as possible but stopping when there is no basis in the image information for following the object further. The method was tested on a set of synthetic images of tubes of varying curvature and torsion that gradually narrow to a radius of zero. Varying amounts of image noise were added. In none of the images did the core continue following past where the tube came to a point. In $87 \%$ of the cases the core stopped within one voxel of the actual end of the tube. In the remaining $13 \%$ of the cases the core stopped between two and five voxels early.

\subsection{Results in Clinical Images}

Core following with branch handling and core termination was tested on a clinical MRA image of the head acquired using a Siemens 3T system. The image is 512 x 512 $\mathrm{x} 91$ voxels with a resolution of $0.42 \times 0.42 \times 1.25 \mathrm{~mm}$. Figure 5 shows the MRA and the corresponding 3D cores model automatically extracted from the MRA.

The core following algorithm was manually initialized in three places - the left and right carotids and the basilar artery. These three arteries are clearly visible at the bottom of the coronal view of the model. From these three initializations branch handling and core termination were employed without further user interaction to achieve the results shown. Additional validation will be carried out in future work using a more 
complete set of synthetic images, a wider range of clinical images of the head, and clinical data from other parts of the body.
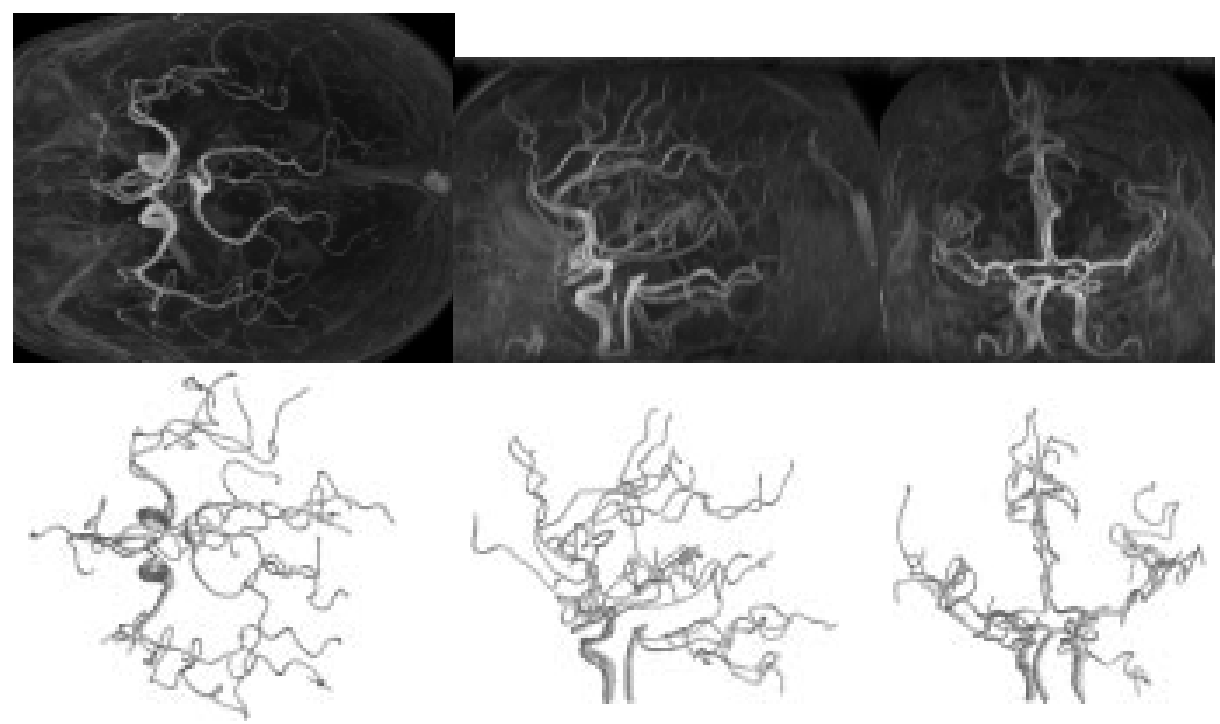

Fig. 5. Maximum intensity projection images of a 389 x 300 x 91 voxel portion of an MRA of the head (top) and corresponding 3D cores model (bottom) shown from axial (left), sagittal (middle), and coronal (right) viewpoints.

\section{Conclusions}

This paper describes the use of cores for segmenting branching tubular structures in three-dimensional grayscale images, with a focus on branches. From initial testing, the method described shows promise for extracting branching objects. For the case of cerebral vasculature, results are encouraging, but branch handling occasionally fails in cases where image noise is high and the widths of the vessels of interest approach the inner scale of the data. A secondary contribution of this paper is automatic core termination in objects that do not have an explicit end. The method described effectively traverses small breaks in the object or small areas of weak image information without losing track of the object. The core termination method has shown good results on a variety of images, although it cannot handle the extreme level of noise that the basic tubular core following method can handle.

Acknowledgements. We are grateful to Russell M. Taylor II for driving problems and helpful discussions and to James Damon for mathematical insights into our problem. We also acknowledge Carl-Fredrik Westin and Raul Estepar, Brigham and Women's Hospital, for their gracious help in image filtering. This work was done 
under the support of ONR MURI grant N00014-98-1-0597 as well as the partial support of NIH grants R01 EB000219 NIBIB, R01 HL69808 NIHLB, and P01 CA47982 NCI.

\section{References}

1. Bullitt, E, SR Aylward, K Smith, S Mukherji, M Jiroutek, K Muller (2001). Symbolic description of intracerebral vessels segmented from magnetic resonance angiograms and evaluation by comparison with X-ray angiograms. Medical Image Analysis, 5: 157-169.

2. Fritsch, DS, D Eberly, SM Pizer, MJ McAuliffe (1995). Stimulated cores and their applications in medical imaging. Information Processing in Medical Imaging, Y Bizais, C Barillot, R DiPaola, eds., Kluwer Series in Computational Imaging and Vision: 365-368.

3. Furst, JD (1999). Height Ridges of Oriented Medialness. Ph.D. Dissertation, Department of Computer Science, University of North Carolina at Chapel Hill.

4. Morse, BS, SM Pizer, DT Puff, C Gu (1998). Zoom-invariant vision of figural shape: effects on cores of image disturbances. Computer Vision and Image Understanding, 69: 7286.

5. Pizer, SM, D Eberly, BS Morse, DS Fritsch (1998). Zoom-invariant vision of figural shape: The mathematics of cores. Computer Vision and Image Understanding, 69: 55-71.

6. Frangi, AF, WJ Niessen, RM Hoogeveen, T van Walsum, MA Viergever (1999). Modelbased quantitation of 3D magnetic resonance angiographic images. IEEE Transactions on Medical Imaging, 18: 946-956.

7. Lorigo, LM, O Faugeras, WEL Grimson, R Keriven, R Kikinis, CF Westin (1999). Codimension 2 geodesic active contours for MRA segmentation. Information Processing in Medical Imaging, A Kuba, M Sámal, A Todd-Pokropek, eds., Lecture Notes in Computer Science, 1613: 126-139.

8. Vasilevskiy, A, K Siddiqi (2002). Flux maximizing geometric flows. IEEE Transactions on Pattern Analysis and Machine Intelligence, 24: 1565-1578.

9. Aylward, SR, E Bullitt (2002). Initialization, noise, singularities, and scale in height ridge traversal for tubular object centerline extraction. IEEE Transactions on Medical Imaging, 21: $61-75$.

10. Aylward, SR, SM Pizer, E Bullitt, D Eberly (1996). Intensity ridge and widths for tubular object segmentation and description. IEEE Workshop on Mathematical Methods in Biomedical Image Analysis, 56: 131-138.

11. Miller, JE (1998). Relative Critical Sets and their Application to Image Analysis. Ph.D. Dissertation, Department of Mathematics, University of North Carolina at Chapel Hill.

12. Westin, CF, L Wigstrom, T Loock, L Sjoqvist, R Kikinis, H Knutsson (2001). Threedimensional adaptive filtering in magnetic resonance angiography. Journal of Magnetic Resonance Imaging, 14: 63-71.

13. Witkin, AP (1983). Scale-space filtering. Proceedings of the Eight International Joint Conference on Artificial Intelligence: 1019-1022.

14. Eberly, D (1996). Ridges in image and data analysis. Computational Imaging and Vision Series. Kluwer Academic Publishers, Dordrecht, Netherlands, 1996.

15. Blom, J (1991). Affine invariant corner detection. Ph.D. Thesis, Utrecht University.

16. Lindeberg, T (1994). Scale-Space Theory in Computer Vision. Kluwer Academic Publishers, Dordrecht, Netherlands.

17. ter Haar Romeny, BM (2002). Front-end vision and multi-scale image analysis. Kluwer Academic Publishers. 